\title{
Study Protocol Uniform Resource Locator Description
}

National Cancer Institute

\section{Source}

National Cancer Institute. Study Protocol Uniform Resource Locator Description. NCI

Thesaurus. Code C127802.

A textual description for the uniform resource locator of a clinical study protocol. 